University of Montana

ScholarWorks at University of Montana

\title{
Life Stage Simulation Analysis: Estimating Vital-Rate Effects on Population Growth for Conservation
}

\author{
M. J. Wisdom \\ L. Scott Mills \\ University of Montana - Missoula, Iscott.mills@umontana.edu \\ D. F. Doak
}

Follow this and additional works at: https://scholarworks.umt.edu/wildbio_pubs

Part of the Life Sciences Commons

Let us know how access to this document benefits you.

\section{Recommended Citation}

Wisdom, M. J.; Mills, L. Scott; and Doak, D. F., "Life Stage Simulation Analysis: Estimating Vital-Rate Effects on Population Growth for Conservation" (2000). Wildlife Biology Faculty Publications. 11.

https://scholarworks.umt.edu/wildbio_pubs/11

This Article is brought to you for free and open access by the Wildlife Biology at ScholarWorks at University of Montana. It has been accepted for inclusion in Wildlife Biology Faculty Publications by an authorized administrator of ScholarWorks at University of Montana. For more information, please contact scholarworks@mso.umt.edu. 


\title{
LIFE STAGE SIMULATION ANALYSIS: ESTIMATING VITAL-RATE EFFECTS ON POPULATION GROWTH FOR CONSERVATION
}

\author{
Michael J. Wisdom,,$^{1,4}$ L. Scott Mills, ${ }^{2}$ And Daniel F. DoaK ${ }^{3}$ \\ ${ }^{1}$ Department of Fish and Wildife Resources, University of Idaho, Moscow, Idaho 83844 USA \\ ${ }^{2}$ Wildlife Biology Program, School of Forestry, University of Montana, Missoula, Montana 59812 USA \\ ${ }^{3}$ Department of Environmental Studies, University of California, Santa Cruz, California 95064 USA
}

Abstract. We developed a simulation method, known as life-stage simulation analysis (LSA) to measure potential effects of uncertainty and variation in vital rates on population growth $(\lambda)$ for purposes of species conservation planning. Under LSA, we specify plausible or hypothesized levels of uncertainty, variation, and covariation in vital rates for a given population. We use these data under resampling simulations to establish random combinations of vital rates for a large number of matrix replicates and finally summarize results from the matrix replicates to estimate potential effects of each vital rate on $\lambda$ in a probabilitybased context. Estimates of potential effects are based on a variety of summary statistics, such as frequency of replicates having the same vital rate of highest elasticity, difference in elasticity values calculated under simulated conditions vs. elasticities calculated using mean invariant vital rates, percentage of replicates having positive population growth, and variation in $\lambda$ explained by variation in each vital rate. To illustrate, we applied LSA to vital rates for two vertebrates: desert tortoise (Gopherus agassizii) and Greater Prairie Chicken (Tympanuchus cupido). Results for the prairie chicken indicated that a single vital rate consistently had greatest effect on population growth. Results for desert tortoise, however, suggested that a variety of life stages could have strong effects on population growth. Additional simulations for the Greater Prairie Chicken under a hypothetical conservation plan also demonstrated that a variety of vital rates could be manipulated to achieve desired population growth. To improve the reliability of inference, we recommend that potential effects of vital rates on $\lambda$ be evaluated using a probability-based approach like LSA. LSA is an important complement to other methods that evaluate vital-rate effects on $\lambda$, including classical elasticity analysis, retrospective methods of variance decomposition, and simulation of the effects of environmental stochasticity.

Key words: demography; Gopherus agassizii (desert tortoise); elasticity; finite rate of increase; life-stage simulation analysis and importance; matrix population models; population growth; sensitivity; species conservation; Tympanuchus cupido (Greater Prairie Chicken); variance and covariance; vital rates.

\section{INTRODUCTION}

The use of matrix population models to address issues of species conservation has increased dramatically in recent years. The surge of interest in matrix models stems from easy-to-use computer packages, the directness with which matrix models are connected to field data, and the clarity with which matrix models link life history traits to population characteristics, such as rate of population growth (finite rate of increase, $\lambda$ ). Consequently, matrix population models are a primary means of assessing population viability for many species of concern (Burgman et al. 1993, Mills et al. 1996, Heppell et al. 2000).

Formal sensitivity analysis of the effect of changes in vital rates on population growth is one of the most

Manuscript received 9 October 1998; revised 16 June 1999; accepted 16 June 1999. For reprints of this Special Feature, see footnote 1, p. 605 .

${ }^{4}$ Present address: U.S. Forest Service, Forestry and Range Sciences Laboratory, 1401 Gekeler Lane, La Grande, Oregon 97850 USA. E-mail: wisdomm@eou.edu rapidly increasing uses of matrix models. One approach, calculating analytical sensitivities and elasticities, is popular, because it provides a clear, straightforward ranking of the importance of different demographic rates using sensitivity and elasticity values (de Kroon et al. 1986, van Groenendael et al. 1988, Caswell 1989, de Kroon et al. 2000).

Because elasticities are calculated easily, and presumably can be interpreted in a straightforward manner, a plethora of recent studies have used elasticities to infer which life stages are most important for species conservation (e.g., Crouse et al. 1987, Wootton and Bell 1992, Brault and Caswell 1993, Crowder et al. 1994, Doak et al. 1994, Escos et al. 1994, Heppell et al. 1994, Olmsted and Alvarez-Buylla 1995, Heppell et al. 1996, Marschall and Crowder 1996, Ratsirarson et al. 1996, Silvertown et al. 1996). However, different forms of real variation (spatial and temporal variation) or perceived variation (sampling and measurement errors) can affect both the rank order of elasticities (Mills et al. 1999), as well as their absolute value (Gotelli 
1991, Kalisz and McPeek 1992, Benton and Grant 1996, Wisdom and Mills 1997, Crooks et al. 1998). Consequently, elasticities calculated from one set of mean, invariant vital rates could be misleading. Furthermore, the vital rates that vary the most tend to be the ones with the smallest elasticities (Brault and Caswell 1983, Gaillard et al. 1998, Pfister 1998). Together, these results suggest that vital-rate uncertainty, variation, and covariation cannot be ignored. Accordingly, approaches such as retrospective methods of variance decomposition (life table response experiments [LTREs]; Caswell 1989, Horvitz et al. 1997) have been used to evaluate past effects of vital-rate variation and covariation on population growth as a complement to classical elasticity analysis (e.g., Brault and Caswell 1993). Effective conservation of imperiled species, however, also requires analysis of a variety of future, potential sources of demographic uncertainty, variation, and covariation on population growth to complement our understanding of past effects.

Consequently, a combination of different analytical approaches may be useful to managers interested in identifying the most "important" life stages for species conservation. This point was demonstrated by Horvitz et al. (1997), who compared and contrasted measures of life stage importance, based on traditional methods of sensitivity and elasticity analysis, with methods based on effects of variance and covariance of vital rates (variance decomposition analysis). Their results illustrated substantial differences in the two methods as measures of life stage importance.

While such new analyses have begun to explore the interpretation of different measures of life stage importance in the face of uncertain and changing vital rates, a more complete set of analytical tools for evaluating life stage importance has yet to be devised. Moreover, the need for integrated, reliable tools for assessing life stage importance for species conservation continues to escalate with the increasing rate at which species are listed as threatened and endangered (Flather et al. 1994). In response to this need, our paper demonstrates how a variety of measures of life stage importance can be estimated in a simulation approach that facilitates a diverse evaluation of the potential effects of vital rates on population growth. Specifically, we present a simulation-based method, life stage simulation analysis (LSA), to estimate potential vital-rate effects on population growth under plausible or hypothesized variation, covariation, and uncertainty in vital rates for a given population.

We developed LSA to answer the following questions about potential effects of vital rates on population growth:

1. To what degree do elasticity values change with variation, covariation, and uncertainty in vital rates, considering observed, plausible, or hypothesized sources of spatial variation in vital rates and the un- certainty that potentially exists in estimates of vital rates?

2. If elasticity values change with variation, covariation, and uncertainty in vital rates, are the changes large enough to cause substantial errors in conservation decisions that might be made from conventional calculation of elasticities made from a single matrix of mean or median vital rates, whose parameter estimates are treated as invariant?

3. What other metrics of life stage importance, such as coefficients of determination ( $r^{2}$ values) and measuring the direct response of $\lambda$ to simulated changes in vital rates, can be generated with the use of LSA, and under what conditions do such metrics have utility in conservation planning?

4. How should results from LSA be interpreted as indices of life stage importance to address species conservation, particularly in regard to retrospective analysis (e.g., LTREs) vs. prospective analysis (e.g., classical elasticity analysis), as defined by Horvitz et al. (1997)?

Answering these questions is fundamental to determining whether certain life stages consistently exert the greatest effect on population growth, and whether any single life stage should receive the greatest management attention in conservation plans. In essence, these questions address the uncertainty associated with making correct management decisions for populations of concern, based on the presumed effects of each vital rate on population growth. To illustrate, we conduct example analyses using LSA for two vertebrate species with different life histories, contrast the results with more traditional methods used to analyze life stage importance, and draw some general conclusions about appropriate applications of life stage metrics for effective conservation of imperiled species.

\section{Methods}

Defining LSA

Life stage simulation analysis (LSA) is a simulation method for analyzing potential effects of vital rates on population growth for purposes of species conservation planning (Wisdom and Mills 1997). LSA employs characteristics of both prospective and retrospective forms of matrix perturbation analysis. Prospective analysis was defined by Horvitz et al. (1997) as being an evaluation of life stage importance that addresses potential effects of future, hypothetical changes in vital rates on population growth. This is in contrast to a retrospective analysis, which Horvitz et al. (1997) defined as an evaluation of life stage importance that measures the past response of population growth to observed variation and covariation in vital rates.

LSA is prospective because it uses observed or hypothesized sources of variation in vital rates to evaluate potential effects on future population growth, regardless of what effects vital rates may have had on past

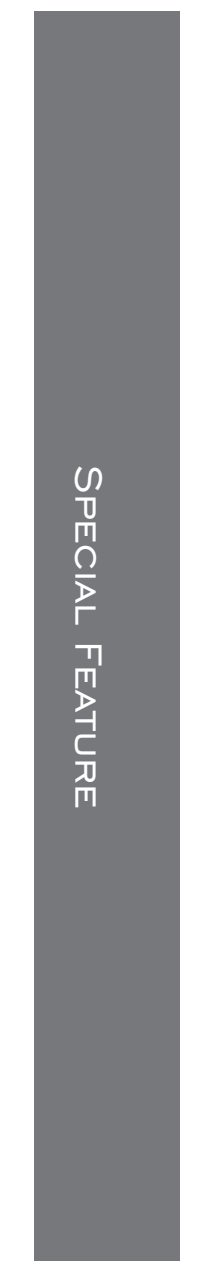


levels of observed population growth. However, LSA also has characteristics of retrospective analysis because empirical sources of vital-rate variation and covariation can be simulated to gain insight about their potential effects on population growth. LSA specifically employs the following analytical steps: (1) vital rates are drawn randomly from a specified probability distribution that reflects the spatial variation and covariation of interest, for a specified population; (2) each set of randomly selected vital rates for the population is used to construct a time-invariant matrix population model (per Gotelli 1991); (3) large numbers of matrix replicates are generated through resampling of the probability distribution of each vital rate for each replicate; and (4) data are analyzed across replicates, using a variety of descriptive statistics, to index the probability of potential effects of each vital rate on $\lambda$, the finite rate of increase.

These analytical steps were designed to complement a variety of established methods that have been used to evaluate vital-rate effects on population growth for species conservation. Established methods include classical elasticity analysis (de Kroon et al. 1986, de Kroon et al. 2000), integrated elasticity analysis (van Tienderen 1995, 2000), retrospective methods of variance decomposition such as LTREs (Caswell 1989, Horvitz et al. 1997), and modeling effects of temporal environmental variation (environmental stochasticity, as defined and used by Tuljapurkar 1990, Benton and Grant 1996, Grant and Benton 2000).

LSA is complementary to classical elasticity analysis because changes in rank order and absolute values of elasticities are summarized across large numbers of matrix replicates. LSA also is complementary to integrated elasticity analysis because the simultaneous correlated effects of multiple vital rates on $\lambda$ can be measured in the context of simultaneous background variation and covariation that is simulated within and among all vital rates. Moreover, LSA is complementary to traditional retrospective methods of variance decomposition (Horvitz et al. 1997) because LSA uses regression or related variance reduction techniques to estimate the simulated contribution of future hypothesized variation and covariation in vital rates on population growth, in the context of potential conservation plans. This role of LSA is in contrast to traditional retrospective forms of variance decomposition, such as LTREs, which measure the contribution of past, empirical levels of vital-rate variation and covariation to past changes in $\lambda$.

Finally, LSA is complementary to modeling approaches that use time-varying procedures (Tuljapurkar 1990, Benton and Grant 1996, Grant and Benton 2000) to explore the importance of vital rates under temporal variation. Unlike methods that evaluate effects of temporal variation, LSA depends on $\lambda$ being calculated at a stable age or stage distribution (as do LTREs), so that it evaluates importance under spatial variation, or under temporal variation on a scale that allows stable age distribution to be achieved. Most studies of life stage importance contain a poorly defined mix of spatial, temporal, sampling, and measurement variation, and LSA can be effectively used to consider how each form of variation affects life stage importance (Mills and Lindberg, in press).

Importantly, LSA and methods that simulate effects of time-based environmental stochasticity are similar, in that population response can be measured directly in terms of the number of iterations that yield positive population growth under simulation. This latter characteristic is uniquely different from classical elasticity analysis or from retrospective methods of variance decomposition, and converges on risk assessment simulation approaches (e.g., Akcakaya and Raphael 1998).

\section{Example applications of LSA}

We applied LSA using demographic data from the desert tortoise (Doak et al. 1994) and Greater Prairie Chicken (Wisdom and Mills 1997) as case examples. These species were selected because they differ markedly in life histories, they are subjects of active conservation, and empirical variation in their vital rates has been synthesized and published (see the Appendix). Demographic estimates for both species are composed of an undefined mix of sampling error, measurement error, and spatial and temporal sources of variation. This latter point is important, because nearly all studies of life stage importance have failed to differentiate between sampling and measurement error vs. spatiotemporal variability in vital rates, and few of these studies have differentiated between spatial vs. temporal variation in vital rates (Mills and Lindberg, in press). Moreover, estimates of correlation among vital rates for both species either are poorly estimated or unavailable, which is typical of most demographic studies. For example, we found that $>95 \%$ of 121 demographic studies published during 1988-1998 in Ecology and Journal of Ecology contained no estimates of correlation among vital rates (M. J. Wisdom and L. S. Mills, unpublished data).

Thus, demographic data available for the desert tortoise and Greater Prairie Chicken are typical of those in which life stage metrics are calculated and applied for species in need of conservation. These conditions are important because they represent a typical level of uncertainty and quality of data under which most conservation efforts are undertaken.

Given this high level of uncertainty in vital-rate estimation, the ill-defined mix of different sources of vital-rate variation, and the dearth of knowledge about correlation among vital rates, we designed two example applications under LSA that focused on how such data uncertainties might affect conclusions that can (or cannot) be reached about life stage importance for future conservation of each species. Application 1 was designed to gain general, background understanding 
about the potential effects of varying levels of uncertainty, variation, and covariation in vital rates on population growth. This is similar to population viability analysis (PVA), where diverse modeling conditions are simulated to gain general understanding of potential effects of a variety of factors on population persistence (Burgman et al. 1993).

Application 2 was designed to demonstrate how results from Application 1 could be used to build and test the effectiveness of a hypothetical conservation plan for a species of concern. Specifically, background insights gained from simulations conducted under Application 1 were used to build and evaluate a hypothetical conservation plan for the Greater Prairie Chicken, a species for which demographic analyses have tangible and urgent application (Soulé and Mills 1998 , Westemeier et al. 1998). Application 2 focused on evaluating the plan's effectiveness by measuring the increase in population growth from direct manipulation of vital rates targeted for improvement.

As part of our applications, we identified three major factors that could be varied under LSA that presumably have strong potential effects on population growth and results of our life stage evaluation of each species: (1) the probability distribution underlying each vital rate; (2) the total variation associated with each vital rate, composed of a mix of sampling error, measurement error, and spatial and temporal variation; and (3) the correlation among vital rates. Accordingly, we tailored our applications to illustrate how LSA can be used to estimate vital-rate effects by varying these factors. This point is important, because a variety of probability distributions, sources of vital-rate variation, and demographic variance-covariance structures can be specified under LSA to evaluate the potential effect of each of these factors on population growth. Consequently, as with traditional applications of PVA (Burgman et al. 1993, Mills et al. 1996), no given set of simulated conditions should be expected to compose a definitive analysis. Rather, a variety of conditions must be simulated to gain insight about overall patterns of vitalrate effects on $\lambda$. Our applications therefore illustrate some of the simulated conditions that may be of interest; however, these applications in and of themselves do not constitute a comprehensive battery of conditions that can and should be evaluated for species conservation planning.

\section{Application 1: simulated elasticity and regression metrics}

Under Application 1, we conducted two simulations for each species to illustrate how LSA can be used to gain general, background understanding about potential effects of vital rates on population growth. We refer to "vital rates" as lower level components of the demographic estimates that underlie the elements of matrix population models (Brault and Caswell 1993; the Appendix), rather than the matrix elements themselves.
The specific method of simulation involved the following steps:

1) A complete set of vital rates were randomly selected, with reproductive rates sampled from a lognormal distribution. All other rates were sampled from a $\beta$-probability distribution, with selection adhering to a prespecified variance-covariance structure among vital rates (see the Appendix).

2) The set of randomly selected vital rates were used to calculate matrix elements of survival, growth, and fertility in a stage-based matrix population model (Caswell 1989, Noon and Sauer 1992; the Appendix).

3) The process was executed 1000 times, resulting in 1000 matrix replicates of vital rates and matrix elements for a given species.

4) $\lambda$ and the lower level elasticities (Caswell 1989, Doak et al. 1994) associated with each vital rate were calculated at a stable-stage distribution for each replicate.

5) Data across replicates were analyzed to estimate effects of each vital rate on $\lambda$.

For comparative purposes, we also calculated lower level elasticities associated with vital rates contained in the estimated mean matrix, as is conventionally done under classical elasticity analysis (de Kroon et al. 1986, Caswell 1989). In our Results and Discussion, lower level elasticities of vital rates (Caswell 1989:135-136) are presented and addressed, in contrast to elasticities of matrix elements, which are neither presented nor discussed.

For both the desert tortoise and the prairie chicken simulations, reproductive rates were sampled from a log-normal distribution, and all other rates were sampled from a $\beta$-distribution, under the assumption of central tendency in the true probability distribution underlying each vital rate. Although any distribution could be used, the choice of these distributions, combined with use of an empirical variance and correlation structure, was intended to minimize the frequency of extreme and potentially unrealistic combinations of vital rates coming together under simulation.

For both species, the full level of estimated empirical variance was used for the first simulation (see the Appendix). By contrast, empirical variance of all vital rates was reduced by $50 \%$ (as done by Doak et al. 1994) for the second simulation, in order to test the potential effect of removing variation that might be due to sampling error or temporal variation. Similarly, we used a prespecified correlation structure derived from empirical data on the desert tortoise for both simulations (Doak et al. 1994:450; the Appendix). A positive correlation among adult rates of survival also was specified for the two prairie chicken simulations (see the Appendix).

For both species, estimates of vital-rate effects on $\lambda$ were based on calculation of a variety of descriptive statistics, such as frequency of replicates having the same vital rate of highest elasticity, difference in elas-

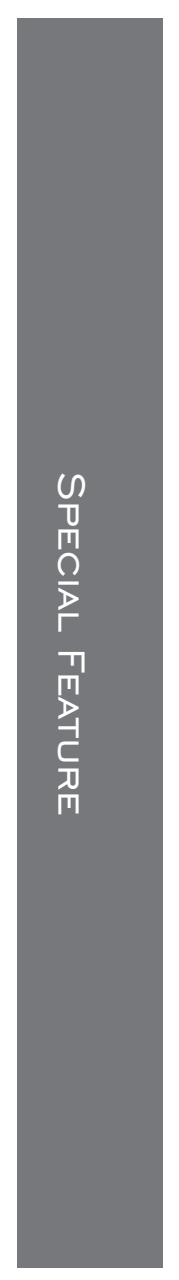


ticity values calculated under simulated conditions vs. elasticities calculated using the mean matrix, percentage of replicates having positive population growth, and coefficients of determination ( $r^{2}$ values), which were calculated by regressing $\lambda$ on each vital rate. We also calculated mean elasticities across all replicates whose vital rate of highest elasticity differed frequently from that of the mean matrix. This allowed us to evaluate whether demographic conditions that led to shifts in the vital rate of highest elasticity reverberated into major changes in the rank order and absolute values of elasticities among all vital rates.

\section{Application 2: simulated population response to direct manipulation of vital rates}

Application 2 addressed the plausible effects of vitalrate improvements that could be accomplished as part of a hypothetical conservation plan for Greater Prairie Chickens. Results from Application 1 were used to identify the vital rates having high, moderate, and low effect on population growth. We then tested the hypothesis that increasing the mean and reducing the variance in egg survival $\left(s_{1_{a}}\right)$ and brood survival $\left(s_{1_{b}}\right)$ (see the Appendix, Table A3) would result in a larger increase in $\lambda$, compared to a similar, composite improvement in the early, mature, and late stages of adult survival $\left(s_{2}-s_{4}\right.$; Table A3). Prior results suggested that such changes in the means and variances would result in the greatest effect on population growth.

This hypothesis was tested with as much realism in terms of conservation management as possible. Key assumptions were as follows:

1 Past variation in vital rates would likely occur under future conservation, unless specific management efforts could be feasibly implemented to reduce variation and increase mean or median values of vital rates.

2 Managers can simultaneously reduce the variance and increase the mean of one or more vital rates as part of a comprehensive strategy to recover an imperiled population.

3 Some vital rates or life stages have high potential for improvement in their parameters with active management, while other life stages have little potential for management-based improvement. Thus, if prospective analysis is used as a realistic tool for species conservation, the analysis must focus on manipulating life stage parameters that are logistically feasible to improve.

4 Past levels of uncertainty in vital rates must be acknowledged and accounted for in any plausible test of the effects of future conservation on population growth. Thus, any target value for a vital rate must include an appropriate level of uncertainty associated with the inability of managers to achieve this value.

5 Some life stage parameters are highly correlated with parameters of other life stages. Consequently, prospective analysis should consider these effects when empirical evidence or logic suggests that correlations are likely or plausible.

Based on these assumptions and our hypothesis of interest, we conducted five simulations in addition to the baseline simulation of no improvements. The first simulation improved egg and brood survival $\left(s_{1_{a}}\right.$ and $s_{1}$, respectively) by $10 \%$ each, compared to the observed means, with $20 \%$ reductions in variation about the means (see the Appendix, Table A3). The second simulation improved the early, mature, and late stages of adult survival $\left(s_{2}-s_{4}\right)$ by $10 \%$ each, with $20 \%$ reductions in variation about the means. The third simulation improved $s_{2}-s_{4}$ by $20 \%$ each, with $20 \%$ reductions in variation about the means.

The fourth and fifth simulations included a correlation structure for specific vital rates. Specifically, the fourth simulation improved egg and brood survival each by $10 \%$ compared to the observed means, with $20 \%$ reductions in variation about the means (same as in the first simulation), but specified a negative correlation $(r=-0.75)$ between the two rates. A negative correlation between egg and brood survival is plausible for prairie chickens and other North American grouse under the assumption of density dependence among life stages during the first year of life (Bergerud 1988). The fifth simulation improved the early, mature, and late stages of adult survival by $10 \%$ each, with $20 \%$ reductions in variation about the means (same as in the second simulation), but specified a positive correlation ( $r=0.75)$ among the three rates. A positive correlation among adult rates of survival is plausible under the assumption that these rates are subjected to the same environmental effects (Bergerud 1988).

The implicit assumption for all five simulations was that any of the improvements in vital-rate parameters were possible as part of a conservation plan, and that past variation in vital rates would continue to be manifest for vital rates not targeted for improvement. Consequently, as part of all five simulations, we maintained the observed means and variations about these values for all vital rates not targeted for improvement, based on empirical studies conducted to date (Wisdom and Mills 1997; see the Appendix).

We then followed the same resampling procedures as outlined for Application 1, except that vital rates for each replicate were sampled from a uniform probability distribution within the empirical range of each rate (see the Appendix). Use of a uniform distribution, combined with use of empirical background variation in vital rates not targeted for improvement, is a cautionary approach to evaluating the merits of proposed conservation plans; that is, each proposal is evaluated under conditions of high uncertainty regarding managers' ability to achieve desired improvements in vital rates, as well as high uncertainty related to knowledge about future variation in vital rates that are not targeted for improvement.

Notably, analysis procedures for Application 2 dif- 

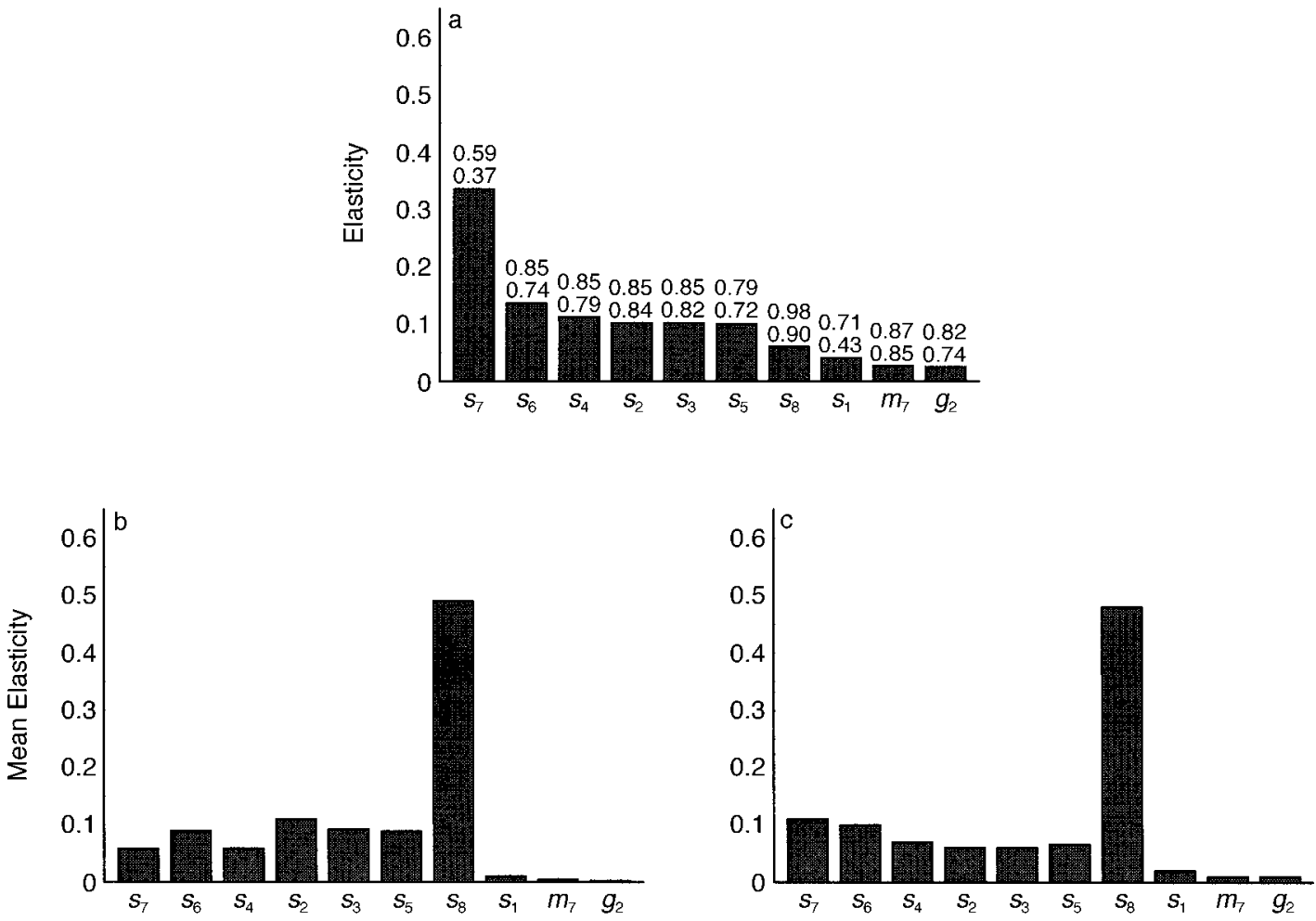

Vital Rate

FIG. 1. Lower level elasticities of vital rates for the desert tortoise, as calculated from (a) the mean invariant vital rates (mean matrix) for the 10 highest elasticities. The numbers above the bars indicate the proportion of times out of 1000 randomly generated matrix replicates that a particular vital rate had an elasticity whose rank order differed from that of the mean matrix. The top set of numbers refers to the proportion for the first simulation (with full variance), and the numbers underneath show deviations in the second simulation (where all vital-rate variations were reduced by $50 \%$ ). When the vital rate of highest elasticity differed from that of the mean matrix, mean elasticities changed considerably for both (b) the first simulation and (c) the second simulation. Vital rates are defined in the Appendix.

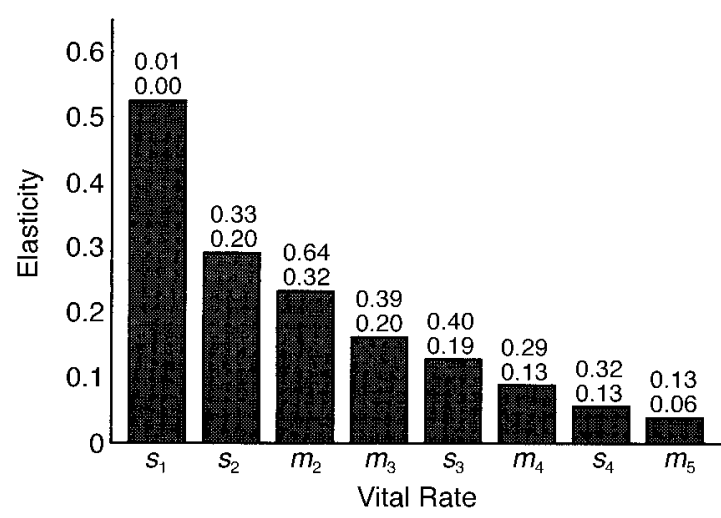

FIG. 2. Lower-level elasticities of vital rates for the Greater Prairie Chicken, as calculated from the mean invariant vital rates (mean matrix). The numbers above the bars indicate the proportion of times out of 1000 randomly generated matrix replicates that a particular vital rate had an elasticity whose rank order differed from that of the mean matrix. The top set of numbers refers to the proportion for the first simulation (with full variance), and the numbers underneath show deviations in the second simulation (where all vital-rate variations were reduced by $50 \%$ ). Vital rates are defined in the Appendix. fered from those of Application 1, because our main interest under Application 2 was to elucidate the merits of each conservation effort (as represented by each of the five simulations), in terms of improving mean population growth and shifting the distribution and range of $\lambda$ values toward positive growth. Analysis for Application 2, therefore, consisted of plotting the distribution and range of $\lambda$ values, calculating the frequency of replicates with $\lambda \geq 1.0$, and calculating mean $\lambda$ for each of the five simulations in relation to these same conditions before vital-rate improvements.

\section{RESULTS}

\section{Application 1: Elasticity and regression metrics}

Classical elasticity values.-Calculations made from the set of mean, invariant vital rates (mean matrix) indicated that highest elasticity was associated with the first stage of adult survival $\left(s_{7}\right)$ for the desert tortoise (Fig. 1a), and with pre-adult survival $\left(s_{1}\right)$ for the Greater Prairie Chicken (Fig. 2). These vital rates would be the targets for management under traditional applications of elasticity analysis. Alternatively, lowest elas-

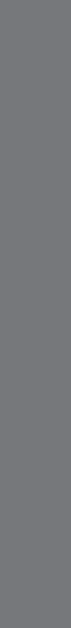



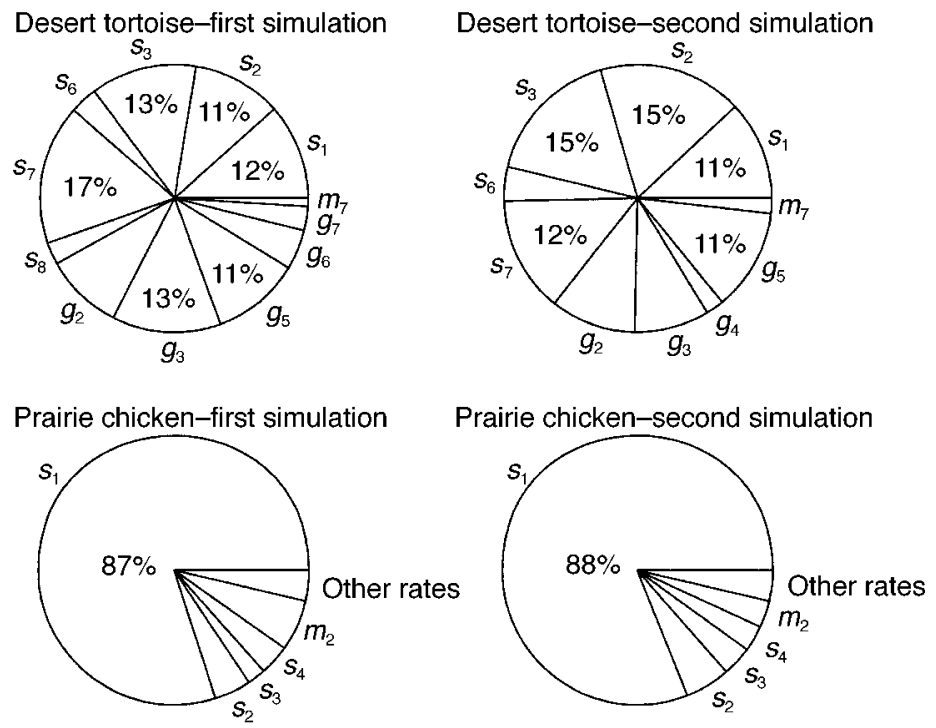

Desert tortoise-second simulation

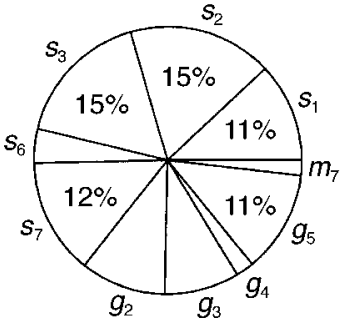

FIG. 3. The variance in population growth explained by each vital rate (coefficients of determination expressed as a percentage on a standardized scale from $0 \%$ to $100 \%$ ) for the desert tortoise and the Greater Prairie Chicken under different methods of simulation, where each simulation was composed of 1000 randomly generated matrix replicates of a matrix population model for each species. The two simulations for each species are described in Figs. 1 and 2. Coefficients of determination were calculated by regressing the finite rate of increase on each randomly varying vital rate across the replicates. Coefficients of determination $\geq 10 \%$ are shown with the associated vital rate. Vital rates not shown accounted for $<1 \%$ of the variation in population growth. Vital rates are defined in the Appendix. ticities were associated with vital rates in all stages of maturation $\left(g_{2}-g_{7}\right)$ and reproduction $\left(m_{6}-m_{8}\right)$ for the desert tortoise, and the late stages of reproduction $\left(m_{4}\right.$ and $m_{5}$ ) and adult survival $\left(s_{3}\right.$ and $s_{4}$ ) for the prairie chicken (Figs. 1a and 2; the Appendix). These results are similar to those reported by Doak et al. (1994) for the desert tortoise and by Wisdom and Mills (1997) for the Greater Prairie Chicken.

Simulations for the desert tortoise.-In contrast to levels of importance implied by the elasticity values calculated using the mean matrix, when variation and covariation in vital rates were considered under simulation, the rank order of vital-rate elasticities for the desert tortoise changed frequently (Fig. 1a). For example, $59 \%$ of the matrix replicates for the desert tortoise simulation under full variation (first simulation), and $37 \%$ of the replicates with variance reduction (second simulation), had a different vital rate of highest elasticity than $s_{7}$, which had the highest elasticity associated with the mean rates. Moreover, the percentage of replicates that had one or more vital rates whose elasticities differed in rank from those of the mean rates ranged from $37 \%$ to $98 \%$ for the two simulations (see Fig. 1a: proportion of simulation replicates that differed from the mean matrix in rank order of each vital-rate elasticity, shown on top of bar charts).

Notably, the inconsistent pattern of elasticity rankings for the desert tortoise was accompanied by extremely large changes in absolute values of the elasticities. For example, $s_{7}$, which had the highest elasticity for the mean matrix $(0.33$; Fig. 1a), had a mean elasticity of only 0.09 (first simulation) and 0.11 (second simulation), across replicates having a vital rate of highest elasticity that was different than $s_{7}$ (Fig. 1b, c). At the same time, $s_{8}$, which had seventh highest elasticity for the mean matrix (0.06; Fig. 1a), had a mean elasticity $>8 \times$ higher $(0.49$; Fig. $1 \mathrm{~b}, \mathrm{c})$ when a rate other than $s_{7}$ was of highest elasticity, which occurred in $37-59 \%$ of the matrix replicates under the two simulations.

Even more notable was the extreme variation in elasticities that occurred for $s_{8}$ under both simulations, despite a $50 \%$ reduction in vital-rate variation that was modeled in the second simulation. Specifically, $s_{8}$ exhibited a bimodal distribution across the 1000 replicates of both simulations, with $>50 \%$ of the matrix replicates having elasticities for $s_{8}$ that were $<0.25$, and $15-25 \%$ additional replicates having elasticities for $s_{8}$ that were $>0.75$.

Variation in population growth that was accounted for by each vital rate also was highly variable for the desert tortoise. No single vital rate accounted for more than $20 \%$ of the variation in $\lambda$ in either simulation, and a wide variety of vital rates accounted for $\geq 10 \%$ of the variation (Fig. 3).

Simulations for the Greater Prairie Chicken.-In contrast to the desert tortoise, the vital rate of highest elasticity for the prairie chicken $\left(s_{1}\right)$ differed in rank from those of the mean matrix for only $1 \%$ of the replicates, for the simulation under full variation (first simulation; Fig. 2). Moreover, elasticity of $s_{1}$ was ranked highest for $100 \%$ of the replicates under variance reduction (second simulation), in full agreement with the mean matrix (Fig. 2). Elasticity rankings of all other vital rates differed more often, however, with changes in rank occurring in 13-64\% of the replicates (see Fig. 2: proportion of simulation replicates on top of bar charts). The vital rate that was associated consistently with the highest elasticity $\left(s_{1}\right)$ also accounted for nearly all $(>85 \%)$ of the variation in population growth of prairie chickens under both simulations (Fig. 3). 


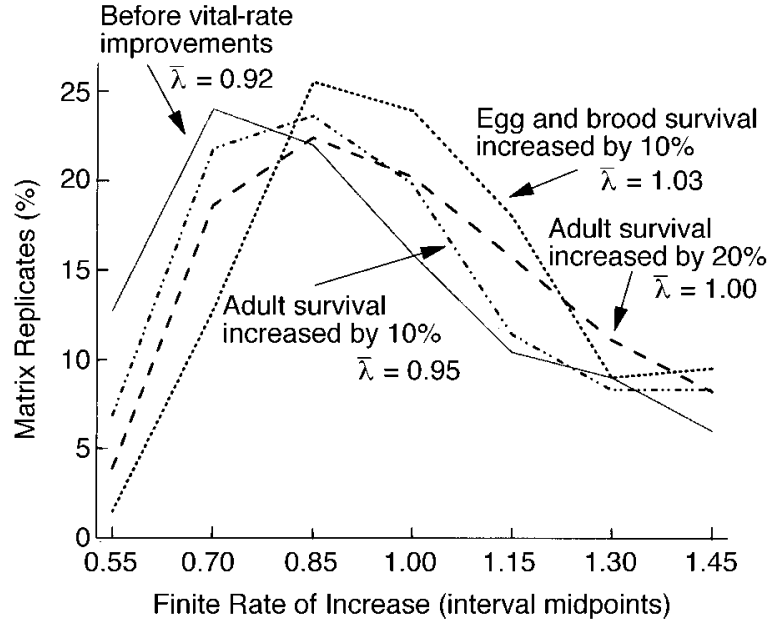

FIG. 4. Distribution and range of the finite rate of increase $(\lambda)$ for Greater Prairie Chickens under four conditions: before vital-rate improvements (—-); after increasing egg and brood survival $\left(s_{1_{a}}\right.$ and $s_{1_{b}}$ ) by $10 \%$, with $20 \%$ reduction in variation (…...); after increasing adult survival $\left(s_{2}-s_{4}\right)$ by $10 \%$, with $20 \%$ reduction in variation $(-\cdots-\cdots-)$; after increasing adult survival by $20 \%$, with $20 \%$ reduction in variation $(----)$. Results are based on analysis methods described for Application 2. Vital rates are defined in the Appendix.

\section{Application 2: Population response to direct manipulation of vital rates}

Potential conservation efforts for the Greater Prairie Chicken changed population growth in interesting ways (Fig. 4). Efforts that would increase mean egg and brood survival $\left(s_{1_{a}}\right.$ and $s_{1_{b}}$ ) by $10 \%$, combined with $20 \%$ reductions in variation around these mean values, caused the mean rate of population growth to increase $11 \%$ (mean $\lambda=0.92$ beforehand, 1.03 afterward). By contrast, simultaneously increasing the mean values of early, mature, and late stages of adult survival $\left(s_{2}-s_{4}\right)$ by $10 \%$, combined with $20 \%$ reductions in variation about these rates, caused mean $\lambda$ to increase only $3 \%$ and remain in decline (mean $\lambda=0.95$ ). However, a larger increase in adult survival by $20 \%$, with the same reduction in variation, caused mean $\lambda$ to increase $8 \%$ and reach a nondeclining value (mean $\lambda=1.00$ ). Moreover, the percentage of replicates with positive population growth was similar when egg and brood survival was increased by $10 \%$ vs. adult survival being increased by $20 \%$ ( $47 \%$ vs. $44 \%$ of replicates), implying that conservation efforts directed at either egg and brood survival, or at adult survival, could reverse the declining rate of mean population growth (Fig. 4). However, the high percentage of replicates with declining population growth for both conservation efforts ( $>50 \%$ of replicates for each conservation effort had a declining growth rate) further suggests that neither improvement in egg and brood survival $\left(s_{1_{a}}\right.$ and $s_{1_{b}}$ ) nor in adult survival $\left(s_{2}-s_{4}\right)$ alone would result in a high probability of achieving increasing population growth under the conditions that were simulated.

Conservation efforts that made similar changes in mean and range of prairie chicken vital rates, but in the context of different correlations among rates, led to disparate patterns of change in population growth (Fig. 5). Increasing mean egg and brood survival by $10 \%$, combined with $20 \%$ reductions in variation around these mean values, but with a negative correlation structure between the two rates $(r=-0.75)$ rates, caused mean $\lambda$ to change from 0.92 beforehand (Fig. 4) to 1.00 afterward (Fig. 5a). However, the increase in mean population growth was not as high under negative correlation of egg and brood survival as occurred without correlation (mean $\lambda=1.00$ with negative correlation, mean $\lambda=1.03$ without correlation; Fig. 5a). Interestingly, while mean $\lambda$ was lower under vital-rate improvements that assumed a negative correlation between egg and brood survival vs. like improvements without correlation, the variability about mean population growth was considerably lower with correlation $(\mathrm{SD}=0.15)$ than without $(\mathrm{SD}=0.25)$. This lower var-

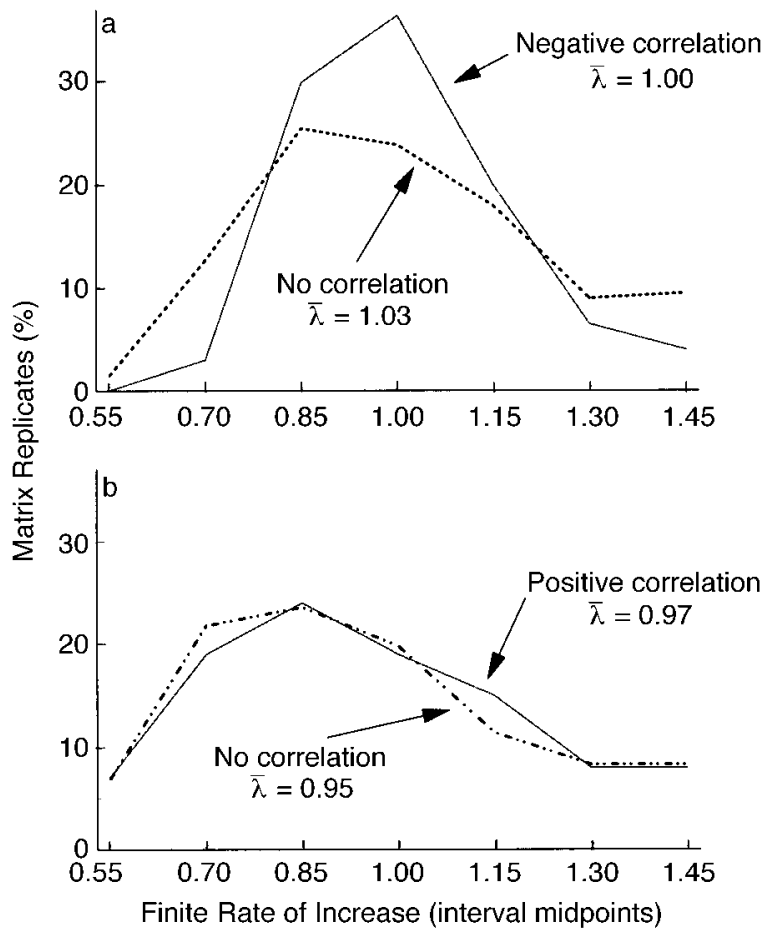

FIG. 5. Distribution and range of the finite rate of increase $(\lambda)$ for Greater Prairie Chickens under four conditions of vitalrate improvements: (a) increasing egg and brood survival $\left(s_{1_{a}}\right.$ and $\left.s_{1_{b}}\right)$ by $10 \%$, with $20 \%$ reduction in variation and either no correlation between egg and brood survival (.........), or negative correlation $(r=-0.75)$ between egg and brood survival (- $)$; (b) increasing adult survival $\left(s_{2}-s_{4}\right)$ by $10 \%$, with $20 \%$ reduction in variation and either no correlation among adult rates of survival $(-\cdots-\cdots-)$, or positive correlation $(r=0.75)$ among adult rates of survival ( - . Results are based on analysis methods described for Application 2. Vital rates are defined in the Appendix. 
iability under negative correlation was illustrated by the higher and more narrow distribution of values about mean population growth, under correlation than without correlation (Fig. 5a).

Finally, conservation efforts to simultaneously increase the values of all stages of adult survival by $10 \%$, combined with $20 \%$ reductions in variation about these rates, with the further assumption of positive correlation $(r=0.75)$ among the rates, caused the mean rate of population growth to increase, as well (mean $\lambda=$ 0.92 beforehand, Fig. 4; 0.97 afterward, Fig 5b). This increase in population growth was nearly the same as that observed for the same vital-rate improvements without correlation (Fig. 5b), and neither set of improvements resulted in stationary or increasing population growth (mean $\lambda=0.95$ without correlation, mean $\lambda=0.97$ with correlation).

\section{DISCUSSION}

\section{Mechanisms and patterns of life stage importance}

Ecological theory related to life history strategies (e.g., Pianka 1970, Southwood et al. 1974) suggests that adult stages of survival are most "important" for persistence of long-lived vertebrates, such as the desert tortoise, and that reproductive and juvenile stages are critical to persistence of short-lived vertebrates such as the Greater Prairie Chicken. Similarly, analytical sensitivities and elasticities of adult survival tend to increase with generation length (Lebreton and Clobert 1991, Gaillard et al. 1998, Heppell et al. 2000, Sæther and Bakke 2000). Our deterministic elasticities from the mean matrix of vital rates for both species support this theory, as elasticities were highest for early adult survival $\left(s_{7}\right)$ for the long-lived desert tortoise, but were highest for juvenile survival $\left(s_{1}\right)$ for the relatively short-lived prairie chicken.

Results from incorporating variation under simulation, however, suggest that mean elasticities alone are not always good predictors of the importance of certain vital rates to population growth (Gaillard et al. 1998, Mills et al. 1999). In the case of the desert tortoise, a wide variety of vital rates appeared to have relatively equal effect on population growth. That is, no single life stage had a notably strong, consistent effect on population growth, as evidenced by the large shifts in rank and value of the simulated elasticities, and by the equally sparse amount of variation in $\lambda$ accounted for by a large number of vital rates.

These shifts in elasticity patterns with variation in vital rates beg the following question: Would these shifts be predicted by life history theory and natural selection? In this context, Pfister (1998) tested the life history prediction that natural selection should minimize variation in population growth rate, where growth rate is considered a surrogate for fitness. She found that variance in population growth rate was in fact minimized, with a strong moderating effect on $\lambda$ caused by an overwhelming negative correlation between sensitivities (or elasticities) and temporal variation of vital rates for the 30 field populations that were examined. There were no cases where both variation in a rate and sensitivity (or elasticity) were simultaneously large for a given matrix element. Given these findings, our results for the prairie chicken are surprising, because one rate, juvenile survival $\left(s_{1}\right)$, had the highest elasticity, the widest variation, and accounted for the vast majority of the variation in growth rate. In contrast, results for the desert tortoise support the theoretical expectation that "life histories appeared to be a suite of demographic traits that minimized variation in population growth rate," (Pfister 1998:217) in that the rates with the highest elasticities (Fig. 1) did not vary the most (see the Appendix), so no single rate explained a preponderance of the variation in $\lambda$ (Fig. 3).

One reason for the fact that results for the prairie chicken were inconsistent with this expectation, and instead had a single rate with both high elasticity and high variation, may relate to spatial variation and sampling error being included with temporal variation (Pfister 1998, Gaillard et al. 1998). Identifying and removing sampling error is important, because different vital rates typically have different absolute amounts of sampling error, as well as different ratios of sampling to process error, which may cloud the true contribution of each vital rate to variance in $\lambda$ (Mills and Lindberg, in press). This issue has been addressed in the context of population viability analysis (Ludwig 1999, White, in press).

Another reason for the contradiction of the prairie chicken results with ecological theory may be the fact that our demographic estimates were largely obtained from populations that have declined in relation to widespread human-caused disturbances and environmental degradation, resulting in large range contractions and significant reductions in size of populations (Johnsgard 1973, Westemeier et al. 1998). These overwhelming anthropogenic sources of environmental degradation may have introduced greater variation in certain life stages than was present over evolutionary time, thus confounding the life history strategy of the species. If this is the case, the life-history context for the species' persistence could be subverted, making such species highly vulnerable to extinction (Pimm et al. 1988).

Finally, this mismatch with evolutionary theory may be complicated by the matrix structure itself (van Groenendael et al. 1988). The choice of an age-based vs. a stage-based model, and the number and type of stages chosen in a stage-based model, affect the calculation of elasticity and regression values (Easterling et al. 2000). Because lower level elasticities can be summed across vital rates (Caswell 1989), some effects of combining ages or stages on elasticity values and rankings can be assessed in a straightforward manner. This analytical flexibility allows different combinations of life 
stages to be evaluated as they relate to both the theory and effectiveness of proposed conservation efforts.

Implications for conservation.-Several of our results suggest the need for greater caution in the assessment of life stage importance for species conservation (also see Ehrlen and van Groenendael 1998, Mills et al. 1999, de Kroon et al. 2000, Grant and Benton 2000, Sæther and Bakke 2000, Benton and Grant 1999). In the case of the desert tortoise, rank order and absolute values of vital-rate elasticities shifted wildly when demographic uncertainty, variation, and covariation were incorporated. This suggests that elasticities based on a single matrix of mean invariant rates could be misleading under conditions of either high variance or high uncertainty in vital rates. Moreover, none of these shifts in range and value of vitalrate elasticities appeared to be mediated by the level of covariation among rates, or by a significant (50\%) reduction in the amount of vital-rate variation that was simulated. Obviously, the probability of correctly choosing the "most important" vital rate for management may be quite low under such conditions.

On the surface, these inconsistent results for the desert tortoise appear to confound identification of clear conservation direction for the species. In particular, the strong shift in rank and value of elasticities between $s_{7}$ and $s_{8}$ (early and late stages of adult survival) under our simulations requires further explanation if conservation direction is to be clarified. In that light, we explored results from the two desert tortoise simulations in more detail and discovered the following: (1) either $s_{7}$ or $s_{8}$ was of highest elasticity for the majority $(>70 \%)$ of the simulation replicates; (2) when $s_{7}$ was of highest elasticity, its mean annual survival was $>0.90$, whereas mean survival for $s_{8}$ was $<0.85$, with an associated mean population growth rate of 0.96 0.99 ; (3) when $s_{8}$ was of highest elasticity, its mean annual survival was $>0.91$, whereas mean survival for $s_{7}$ was $<0.84$, with an associated mean growth rate of 0.92-0.96; and (4) no strong correlations existed between either of these two vital rates with any other vital rates in relation to the large shifts in elasticity values that were observed for $s_{7}$ and $s_{8}$. These additional findings suggest that relatively small amounts of variation in $s_{7}$ and $s_{8}$ are associated with large changes in elasticity values that index meaningful differences in population growth. These findings further suggest that maintenance of mean annual survival at values $>0.90$ for $s_{7}$ is perhaps one important criterion for increasing the mean rate of population growth for the desert tortoise. Finally, our results suggest that both $s_{7}$ and $s_{8}$ are important to conservation of the desert tortoise, and that both stages deserve high research and management attention. However, a comprehensive approach to improve all vital-rate values for the desert tortoise remains the single best solution to population recovery, given the sparse amount of variation in $\lambda$ that was accounted for by any single vital rate.
Results between the two species, under the simulations of Application 1, further illustrated important points for species conservation, with two distinctly different patterns of life stage importance that emerged. In one case, a single vital rate $\left(s_{1}\right)$ typically had greatest effect on population growth (Greater Prairie Chicken), as measured both by elasticity and coefficients of determination. In the other case, both rank order and absolute values of vital-rate elasticities changed frequently and radically (desert tortoise). Moreover, no vital rate accounted for a high amount of variation in $\lambda$ for the desert tortoise under either simulation. Such disparate interspecific differences in patterns of life stage importance deserve additional research attention to gain further insight about the theoretical underpinnings for such differences (per discussion of Benton and Grant 1999), which in turn could help guide development of appropriate conservation efforts.

Results of Application 2 demonstrated how the life stage simulation analysis (LSA) can be used to test the presumed benefits of specific conservation strategies that target particular vital rates for parameter (mean and variance) improvements (also see Citta and Mills [1999]). In the case of the Greater Prairie Chicken, results from the Application 2 suggest that identification of only one life stage as "most important," such as was done by Wisdom and Mills (1997), may be overly simplistic. Instead, Application 2 suggests that at least two different approaches, one emphasizing increases in two components of subadult survival $\left(s_{1_{a}}\right.$ and $s_{1_{b}}$ ) and the other emphasizing increases in adult survival $\left(s_{2}-s_{4}\right)$, could achieve positive effects on mean population growth for species conservation. Which approach is best depends in large part on the logistical, political, and financial feasibility of changing different vital rates (Nichols et al. 1980, Silvertown et al. 1996, Citta and Mills 1999). However, in the case of the prairie chicken, the majority of matrix replicates still had declining rates of population growth for each of the two proposed approaches, further demonstrating the need to test the effectiveness of a variety of proposed conservation efforts. Interestingly, the summed elasticities from the mean matrix for $s_{1}(0.52)$ vs. those for $s_{2}-s_{4}$ (0.48) support the results of Application 2; that is, proportionate improvements in subadult survival will yield a higher expected increase in $\lambda$ than will like improvements in adult survival.

\section{Utility of LSA}

Analytical flexibility.-Our use of LSA illustrates the utility of measuring vital-rate effects on $\lambda$ in a probability-based context. For each species, we specified variation and covariation in vital rates, used resampling simulations to establish random combinations of these vital rates for each of 1000 matrix replicates, and calculated elasticity and regression parameters using a variety of descriptive statistics. This resulted in a diverse output of metrics that index the probability of each vital

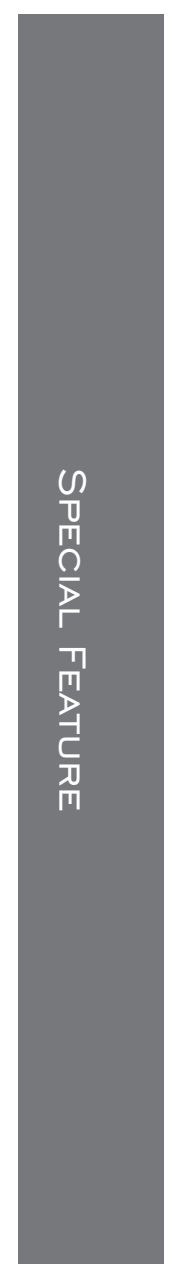


rate's effect on $\lambda$ under the conditions specified. Such an approach, emphasizing variation in vital rates, provides a diverse set of metrics that can complement other established methods that evaluate vital-rate effects on $\lambda$ (also see McCarthy et al. [1995] for a similar regression approach). This use of LSA is similar to error analysis modeling, which is used commonly in other professions, such as economics and industrial quality control, where Monte Carlo methods serve to address the effects of changes (errors) in various model components on changes in desired or predicted outcomes (Sanayei and Saletnik 1996).

Analytical challenges.-LSA has the particular advantage of being able to test the effects of a variety of ecological assumptions on conclusions reached about life stage importance and the response of population growth to changes in vital rates. Among the most critical assumptions are levels of vital-rate uncertainty, variation, and covariation; the probability distribution underlying each vital rate; and the capability to increase vital rates targeted for improvement under a species conservation plan. Unfortunately, empirical data related to these assumptions are absent or sparse for most populations of concern. This latter point is not trivial, as evidenced by the extremely low number of demographic studies reporting simultaneous estimates of demographic variance and covariance. For example, of the 121 studies published during 1988-1998 on population demography that we examined in Ecology and the Journal of Ecology, only four $(<5 \%)$ reported both vital-rate variance and covariance, whereas $>60 \%$ (74 of 121) provided estimates of variance. (Note, however, that variance-covariance structure can still be estimated if studies report separate demographic matrices for different years or locations [e.g., van Tienderen 1995: Methods]). Moreover, most demographic studies have not differentiated between temporal and spatial variation, or between these sources of variation and sampling error (Mills and Lindberg, in press), further complicating the evaluation of life stage importance.

Accordingly, we suggest that a judicious approach would be to evaluate potential effects of a variety of levels and sources of demographic variation (including sampling and measurement error) and covariation. We suggest this mixed strategy because true variance-covariance structure can be poorly estimated due to sampling error or other sampling biases (White, in press); or, in most cases, because variance-covariance structure is not estimated at all. In addition, we suggest that different probability distributions be tested, because the underlying probability distribution of most vital rates is unknown. Finally, due to the many ecological assumptions that are required to conduct LSA and other techniques that evaluate life stage importance, we suggest that LSA be used in concert with other methods, including classical elasticity analysis, integrated elasticity analysis, retrospective forms of variance decom- position, and procedures that evaluate effects of temporal variation.

\section{ACKNOWLEDGMENTS}

We thank H. de Kroon, S. Heppell, E. Garton, M. Groom, B. Johnson, M. Lindberg, J. Peek, C. Pfister, R. Steinhorst, and two anonymous reviewers for their comments. We also thank H. Caswell for dialogue that helped us elucidate the utility of multiple, flexible approaches at evaluating life stage importance. Finally, we thank J. Citta for literature searches and summaries of demographic data related to covariance estimates. Partial support for the work came from NSF-DEB9424566 to D. F. Doak, U.S. Forest Service funding to M. J. Wisdom, and grants from National Science Foundation (NSF) Montanans On a New Track for Science (MONTS) 291835, Boone and Crockett Club, and U.S. Forest Service to L. S. Mills.

\section{Literature Cited}

Akcakaya, H. R., and M. G. Raphael. 1998. Assessing human impact despite uncertainty: viability of the Northern Spotted Owl metapopulation in the northwestern USA. Biodiversity and Conservation 7:875-894.

Benton, T. G., and A. Grant. 1996. How to keep fit in the real world: elasticity analyses and selection pressures on life histories in a variable environment. American Naturalist 147:115-139.

Benton, T. G., and A. Grant. 1999. Elasticity analysis as an important tool in evolutionary and population ecology. Trends in Ecology and Evolution 14:467-471.

Bergerud, A. T. 1988. Population ecology of North American Grouse. Pages 578-777 in A. T. Bergerud and M. W. Gratson, editors. Adaptive strategies and population ecology of northern grouse. Wildlife Management Institute, University of Minnesota Press, Minneapolis, Minnesota, USA.

Brault, S., and H. Caswell. 1993. Pod-specific demography of killer whales (Orcinus orcus). Ecology 74:1444-1454.

Burgman, M. A., S. Ferson, and H. R. Akcakaya. 1993. Risk assessment in conservation biology. Chapman and Hall, New York, New York, USA.

Caswell, H. 1989. Matrix population modeling. Sinauer, Sunderland, Massachusetts, USA.

Caswell, H. 2000. Prospective and retrospective perturbation analyses: their roles in conservation biology. Ecology 81: 619-627.

Citta, J. J., and L. S. Mills. 1999. What do demographic sensitivity analyses tell us about controlling Brown-headed Cowbirds? Studies in Avian Biology 18:121-134.

Crooks, K. R., M. A. Sanjayan, and D. F. Doak. 1998. New insights on cheetah conservation through demographic modeling. Conservation Biology 12:889-895.

Crouse, D. T., L. B. Crowder, and H. Caswell. 1987. A stagebased population model for loggerhead sea turtles and implications for conservation. Ecology 86:1412-1423.

Crowder, L. B., D. T. Crouse, S. S. Heppell, and T. H. Martin. 1994. Predicting the impact of turtle excluder devices on loggerhead sea turtle populations. Ecological Applications 4:437-445.

de Kroon, H., A. Plaisier, J. van Groenendael, and H. Caswell. 1986. Elasticity: the relative contribution of demographic parameters to population growth rate. Ecology 67:14271431.

de Kroon, H., J. van Groenendael, and J. Ehrlén. 2000. Elasticities: a review of methods and model limitations. Ecology 81:607-618.

Doak, D., P. Kareiva, and B. Klepetka. 1994. Modeling population viability for the desert tortoise in the western Mojave Desert. Ecological Applications 4:446-460.

Easterling, M. R., S. P. Ellner, and P. Dixon. 2000. Size- 
specific sensitivity: a new structured population model. Ecology 81:694-708.

Ehrlen, J., and J. van Groenendael. 1998. Direct perturbation analysis for better conservation. Conservation Biology 12: 471-474.

Escos, J., C. L. Alados, and J. M. Emlen. 1994. Application of the stage-projection model with density-dependent fecundity to the population dynamics of Spanish Ibex. Canadian Journal of Zoology 72:731-737.

Flather, C. H., L. A. Joyce, and C. A. Bloomgarden. 1994. Species endangerment patterns in the United States. General Technical Report RM-GTR-241. U.S. Forest Service, Rocky Mountain Research Station, Fort Collins, Colorado, USA.

Gaillard, J., M. Festa-Bianchet, and N. G. Yoccoz. 1998. Population dynamics of large herbivores: variable recruitment with constant adult survival. Trends in Ecology and Evolution 13:58-63.

Gotelli, N. J. 1991. Demographic models for Leptogorgia virgulata, a shallow-water gorgonian. Ecology 72:457467.

Grant, A., and T. G. Benton. 2000. Elasticity analysis for density dependent-populations in stochastic environments. Ecology 81:680-693.

Heppell, S. S., L. B. Crowder, and H. Caswell. 2000. Life histories and elasticity patterns: perturbation analysis for species with minimal demographic data. Ecology 81:654665.

Heppell, S. S., L. B. Crowder, and D. T. Crouse. 1996. Models to evaluate headstarting as a management tool for longlived turtles. Ecological Applications 6:556-565.

Heppell, S. S., J. R. Walters, and L. R. Crowder. 1994. Evaluating management alternatives for Red-cockaded Woodpeckers: a modeling approach. Journal of Wildlife Management 58:479-487.

Horvitz, C. C., D. W. Schemske, and H. Caswell. 1997. The relative "importance" of life history stages to population growth: prospective and retrospective approaches. Pages 247-272 in S. Tuljapurkar and H. Caswell, editors. Structured population models in marine, terrestrial, and freshwater systems. Chapman and Hall, New York, New York, USA.

Johnsgard, P. A. 1973. Grouse and quails of North America. University of Nebraska Press, Lincoln, Nebraska, USA.

Kalisz, S., and M. A. McPeek. 1992. The demography of an age-structured annual: resampled projection matrices, elasticity analyses, and seed bank effects. Ecology 73:10821093.

Lebreton, J. D., and J. Clobert. 1991. Bird population dynamics, management, and conservation: the role of mathematical modeling. Pages 105-125 in C. M. Perrins, J. D. Lebreton, and G. J. M. Hirons, editors. Bird population studies. Oxford University Press, New York, New York, USA.

Ludwig, D. 1999. Is it meaningful to estimate a probability of extinction? Ecology 80:298-310.

Marschall, E. A., and L. B. Crowder. 1996. Assessing population responses to multiple anthropogenic effects: a case study with brook trout. Ecological Applications 6:152-167.

McCarthy, M. A., M. A. Burgman, and S. Ferson. 1995. Sensitivity analysis for models of population viability. Biological Conservation 73:93-100.

Mills, L. S., D. F. Doak, and M. J. Wisdom. 1999. Reliability of conservation actions based on sensitivity analysis of matrix models. Conservation Biology 13:1-15.

Mills L. S., S. G. Hayes, C. Baldwin, M. J. Wisdom, J. Citta, D. J. Mattson, and K. Murphy. 1996. Factors leading to different viability predictions for a grizzly bear data set. Conservation Biology 10:863-873.

Mills L. S., and M. Lindberg. In press. Sensitivity analysis as a means of prioritizing conservation actions. In $\mathrm{S}$. R. Beissinger and D. R. McCullough, editors. Population variability analysis. University of Chicago Press, Chicago, Illinois, USA.

Nichols, J. D., G. L. Hensler, and P. W. Sykes, Jr. 1980. Demography of the Everglade Kite: implications for population management. Ecological Modelling 9:215-232.

Noon, B. R., and J. R. Sauer. 1992. Population models for passerine birds: structure, parameterization, and analysis. Pages 441-464 in D. R. McCullough and R. Barrett, editors. Wildlife 2001: populations. Elsevier Applied Sciences, New York, New York, USA.

Olmsted, I., and E. R. Alvarez-Buylla. 1995. Sustainable harvesting of tropical trees: demography and matrix models of two palm species in Mexico. Ecological Applications 5: 484-500.

Pfister, C. A. 1998. Patterns of variance in stage-structured populations: evolutionary predictions and ecological implications. Proceedings, National Academy of Sciences USA 95:213-218.

Pianka, E. R. 1970. On $r$ and $K$ selection. American Naturalist 104:592-597.

Pimm, S. L., H. L. Jones, and J. Diamond. 1988. On the risk of extinction. American Naturalist 132:757-785.

Ratsirarson, J., J. A. Silander, Jr., and A. F. Richard. 1996. Conservation and management of a threatened Madagascar palm species, Neodypsis decaryi, Jumelle. Conservation Biology 10:40-52.

Sæther, B., and Ø. Bakke. 2000. Avain life history variation and contribution of demographic traits to the population growth rate.. Ecology 81:642-653.

Sanayei, M., and M. J. Saletnik. 1996. Error sensitivity analysis: parameter estimation of structures from static strain measurements, part two. Journal of Structural Engineering 122:563-573.

Silvertown, J., M. Franco, and E. Menges. 1996. Interpretation of elasticity matrices as an aid to the management of plant populations for conservation. Conservation Biology 10:591-597.

Soulé, M. E., and L. S. Mills. 1998. No need to isolate genetics. Science 282:1658-1659.

Southwood, T. R. E., R. M. May, M. P. Hassell, and G. R. Conway. 1974. Ecological strategies and population parameters. American Naturalist 108:791-804.

Tuljapurkar, S. 1990. Population dynamics in variable environments. Springer-Verlag, New York, New York, USA.

van Groenendael, J., H. de Kroon, and H. Caswell. 1988. Projection matrices in population biology. Trends in Ecology and Evolution 3:264-269.

van Tienderen, P. H. 1995. Life cycle trade-offs in matrix population models. Ecology 76:2482-2489.

van Tienderen, P. H. 2000. Elasticities and the link between demographic and evolutionary dynamics. Ecology 81:666679.

Westemeier, R. L., J. D. Brawn, S. A. Simpson, T. L. Esker, R. W. Jansen, J. W. Walk, E. L. Kershner, J. L. Bouzat, and K. N. Paige. 1998. Tracking the long-term decline and recovery of an isolated population. Science 282:16951698.

White, G. C. In press. Population viability analysis: data requirements and essential analyses. In L. Boitani and T. K. Fuller, editors. Research techniques in animal ecology: uses and misuses. Columbia University Press, New York, New York, USA.

Wisdom, M. J., and L. S. Mills. 1997. Sensitivity analysis to guide population recovery: prairie chickens as an example. Journal of Wildlife Management 61:302-312.

Wootton, T. J., and D. A. Bell. 1992. A metapopulation model of the Peregrine Falcon in California: viability and management strategies. Ecological Applications 2:307-321. 


\section{APPENDIX}

In this Appendix, we discuss the vital rates used in stagespecific matrix populations models for the desert tortoise and Greater Prairie Chicken, on which life stage simulation analysis was conducted.

\section{Desert TORTOISE (Gopherus AGASSIZII)}

Our estimates of vital rates for desert tortoise are from Doak et al. (1994), which consist of yearly rates of survival $\left(s_{x}\right)$, growth $\left(g_{x}\right)$, and reproduction $\left(m_{x}\right)$. For estimates of annual survival and growth, we used the means and standard deviations provided in Table 3 of Doak et al. Growth $\left(g_{x}\right)$ is the probability, conditional on survival, of moving from one size class to next larger class (Doak et al. 1994:448-449). For estimates of reproduction $\left(m_{x}\right)$, defined as annual production of female yearlings per breeding female, we calculated the mean and standard deviation of the four values provided by Doak et al. (1994): Table 3.

These rates of $s_{x}, g_{x}$, and $m_{x}$ were derived from data collected among eight sample sites spanning 6-13 yr of study. Because the rates were derived across multiple sites and years, these data contain an undefined mix of spatial and temporal variation, in addition to an unknown amount of sampling and measurement error. Most studies of life stage importance contain a poorly defined mix of spatial, temporal, sampling, and measurement variation (Mills and Lindberg, in press). In this case, we used this mix of variation to test potential effects of vital-rate variation on population growth, and did so by manipulating the total amount of this variation as part of our simulations.

In matrix construction, we follow Doak et al. (1994): Table 5, using eight size classes (Doak et al. 1994: Table 2), with the matrix elements for continued presence in class $x$ (diagonal elements of matrix) equal to $s_{x}\left(1-g_{x}\right)$, and the probability of making a transition to a larger class (subdiagonal elements of matrix) equal to $s_{x} g_{x}$. The first row of reproductive elements are equal to reproductive rates, because these rates are calculated as reproduction of individuals surviving the yearling stage. Note that while Doak et al. (1994) denote the eight size classes as $0-7$, we begin numbering classes at one, and thus refer to classes 1-8. The means (and standard deviations) of the vital rates that underlie the matrix are given in Table A1.

The matrix of dimension $8 \times 8$ was constructed from these vital rates as follows:

$\left[\begin{array}{cccccccc}0 & 0 & 0 & 0 & 0 & m_{6} & m_{7} & m_{8} \\ s_{1} & s_{2}\left(1-g_{2}\right) & 0 & 0 & 0 & 0 & 0 & 0 \\ 0 & s_{2} g_{2} & s_{3}\left(1-g_{3}\right) & 0 & 0 & 0 & 0 & 0 \\ 0 & 0 & s_{3} g_{3} & s_{4}\left(1-g_{4}\right) & 0 & 0 & 0 & 0 \\ 0 & 0 & 0 & s_{4} g_{4} & s_{5}\left(1-g_{5}\right) & 0 & 0 & 0 \\ 0 & 0 & 0 & 0 & s_{5} g_{5} & s_{6}\left(1-g_{6}\right) & 0 & 0 \\ 0 & 0 & 0 & 0 & 0 & s_{6} g_{6} & s_{7}\left(1-g_{7}\right) & 0 \\ 0 & 0 & 0 & 0 & 0 & 0 & s_{7} g_{7} & s_{8}\end{array}\right]$

Except for reproductive rates, all vital rates used in our tortoise simulations are simple probabilities; hence, we se-
TABLE A2. Mean correlation coefficients with $E$, from Doak et al. (1994: Table 4), for the desert tortoise.

\begin{tabular}{clccc}
\hline \hline Stage & \multicolumn{1}{c}{ Class } & $\begin{array}{c}\text { Survival, } \\
s\end{array}$ & $\begin{array}{c}\text { Growth, } \\
g\end{array}$ & $\begin{array}{c}\text { Repro- } \\
\text { duction, } \\
m\end{array}$ \\
\hline 1 & Yearling & 0.750 & $\ldots$ & $\ldots$ \\
2 & Early juvenile & 0.750 & 0.000 & $\ldots$ \\
3 & Late juvenile & 1.000 & 0.379 & $\ldots$ \\
4 & Early immature & -0.269 & 0.202 & $\ldots$ \\
5 & Late immature & -0.443 & 0.651 & $\ldots$ \\
6 & Subadult & 0.367 & 0.548 & 0.000 \\
7 & Early adult & 0.365 & -0.688 & 0.000 \\
8 & Late adult & -0.135 & $\ldots$ & 0.000 \\
\hline
\end{tabular}

Note: Mean coefficients for $s_{1}$ and $s_{2}$ were not given by Doak et al. (1994), and were assumed to be 0.75 .

lected vital rates as random draws from a $\beta$-distribution, using the means and standard deviations to establish each randomly generated matrix replicate under the simulations. Reproductive rates were selected from a log-normal distribution. For the second simulation of Application 1, which specified a $50 \%$ reduction in vital-rate variation, we reduced our standard deviations by $50 \%$.

For all simulations, random draws of vital rates were constrained to the mean correlation structure among vital rates that was derived by Doak et al. (1994:450, Table 4). Under Doak et al.'s analysis, all vital rates were correlated with a single environmental variable, $E$, that fluctuated randomly each year with no autocorrelation. (See Doak et al [1994] for details regarding methods used to generate these correlations.) Mean correlation coefficients with $E$, from Doak et al. (1994): Table 4, are listed in Table A2. Mean coefficients for $s_{1}$ and $s_{2}$ were not given by Doak, and were assumed to be 0.75 . Our approximation of this correlation structure resulted in simulated correlation coefficients that generally were \pm 0.10 of these mean values.

\section{Greater Prairie Chicken (TyMPanuChus CuPido)}

Demographic estimates for the Greater Prairie Chicken were adapted from Wisdom and Mills (1997). Specifically, mean and range of vital rates are from Wisdom and Mills (1997):304-305, Tables 1 and 2, and standard deviations are based on data from the demographic studies cited on pages 305-307, therein. These demographic estimates were synthesized from multiple studies and populations, with each study spanning different time periods. Consequently, these estimates contain an undefined mix of spatial and temporal variation, in addition to an unknown amount of sampling and measurement error, which is typical of most studies of life stage importance that have been conducted to date (Mills and Lindberg, in press). Because our goal was to test potential effects of different amounts of vital-rate variation, we simply

TABle A1. Means (and standard deviations) of the vital rates that underlie the matrix for the desert tortoise.

\begin{tabular}{clccc}
\hline \hline Stage & \multicolumn{1}{c}{ Class } & Survival, $s$ & Growth, $g$ & Reproduction, $m$ \\
\hline 1 & Yearling & $0.716(0.232)$ & $\ldots$ & $\ldots$ \\
2 & Early juvenile & $0.716(0.232)$ & $0.208(0.268)$ & $\ldots$ \\
3 & Late juvenile & $0.716(0.232)$ & $0.208(0.268)$ & $\ldots$ \\
4 & Early immature & $0.839(0.176)$ & $0.280(0.158)$ & $\ldots$ \\
5 & Late immature & $0.785(0.147)$ & $0.287(0.261)$ & $\ldots$ \\
6 & Subadult & $0.927(0.071)$ & $0.269(0.187)$ & $0.996(0.972)$ \\
7 & Early adult & $0.867(0.129)$ & $0.018(0.370)$ & $1.530(1.470)$ \\
8 & Late adult & $0.860(0.123)$ & $\ldots$ & $1.927(1.950)$ \\
\hline
\end{tabular}


TABLE A3. Mean rates (1 SD; ranges) derived for stages and component classes for the Greater Prairie Chicken.

\begin{tabular}{lllc}
\hline \hline Stage & \multicolumn{1}{c}{ Class } & \multicolumn{1}{c}{ Survival $(s)$} & Reproduction $(m)$ \\
\hline 1 & Pre-adult & $s_{1_{a}} \cdot s_{1_{b}} \cdot s_{1_{c}}$ & $\ldots$ \\
$1 a$ & Egg, 1 mo & $0.500(0.156 ; 0.200-0.800)$ & $\ldots$ \\
$1 b$ & Brood, 3 mo & $0.350(0.122 ; 0.150-0.550)$ & $\ldots$ \\
$1 c$ & Postbrood, 8 mo & $0.540(0.108 ; 0.540-0.690)$ & $\ldots$ \\
2 & Early adult & $0.445(0.081 ; 0.380-0.510)$ & $4.381(1.055 ; 2.803-5.960)$ \\
3 & Mature adult & $0.510(0.079 ; 0.450-0.570)$ & $6.382(0.945 ; 4.914-7.850)$ \\
4,5 & Late adult & $0.284(0.090 ; 0.172-0.396)$ & $6.382(0.945 ; 4.914-7.850)$ \\
\hline
\end{tabular}

used these estimates as representing the typical level of quality of data used to evaluate life stage importance, and manipulated the total amount of this variation as part of our simulations.

Wisdom and Mills (1997) parameterized vital rates for eight age classes into a Leslie matrix using a postbirth pulse formulation (Caswell 1989). Wisdom and Mills (1997) also partitioned the first age class, age 0 survival, into three components: egg survival (nest success), brood survival, and postbrood survival. We collapsed these eight age classes into four stages of a Lefkovitch matrix using a postbirth pulse formulation (Caswell 1989). The first three stages of our model equal the first three age classes of Wisdom and Mills (1997), including the three components of age 0 survival. The fourth and last stage is a generalization of the last five age classes of Wisdom and Mills (1997), where the stage-based estimates represent the grand mean of the lower and upper limits of the mean values of vital rates, calculated among the five age classes. Table A3 shows the mean rates, standard deviations, and ranges that we derived for these stages and components.

The matrix of dimension $4 \times 4$ was constructed from these vital rates as follows:

$$
\left[\begin{array}{cccc}
s_{1} m_{2} & s_{2} m_{3} & s_{3} m_{4} & s_{4} m_{5} \\
\left(s_{1_{a}}\right)\left(s_{1_{b}}\right)\left(s_{1_{c}}\right) & 0 & 0 & 0 \\
0 & s_{2} & 0 & 0 \\
0 & 0 & s_{3} & s_{4}
\end{array}\right] .
$$

Note that Wisdom and Mills (1997) used the following notation for their age classes: survival of the first age class was denoted as $S_{0}$ (age-0 survival), second age class as $S_{1}$, and so on. Reproduction of the first age class, postbirth pulse, was denoted as $R_{1}$, second age class as $R_{2}$, and so on. Thus, the notation used for the first three age classes of Wisdom and Mills $\left(S_{0}-S_{2}\right.$ and $\left.R_{1}-R_{3}\right)$ are equivalent to our stages of $s_{1}-s_{3}$ and $m_{2}-m_{4}$. The last five age classes of Wisdom and Mills, $\left(S_{3}-S_{7}\right.$ and $\left.R_{4}-R_{8}\right)$ represent the age classes that were collapsed into the final stages $\left(s_{4}\right.$ and $m_{5}$ ) here.
Except for reproductive estimates, vital rates for all prairie chicken simulations under Application 1 were selected as random draws from a $\beta$-distribution, using the means and standard deviations in Table A3, to establish each randomly generated matrix replicate. Reproductive rates were selected randomly from a log-normal distribution. For the second simulation of Application 1, which specified a 50\% reduction in vital-rate variation, we reduced the standard deviations in Table A 3 by $50 \%$.

For all prairie chicken simulations under Application 1, random draws of vital rates were constrained to a prespecified mean correlation structure among vital rates, using methods of Doak et al. (1994:450, Table 4). Under Doak et al.'s approach, all vital rates were correlated with a single environmental variable, $E$, that fluctuated randomly each year with no autocorrelation. Under this approach, we specified correlation coefficients of 0.75 with $E$ for all rates of adult survival $\left(s_{2}-s_{4}\right)$. Coefficients for all other rates in relation to $E$ were assigned a value of zero.

We also conducted five additional simulations for Application 2 that changed the above vital rates in the following ways: (1) mean values of egg and brood survival $\left(s_{1_{a}}\right.$ and $s_{1_{b}}$ ) were increased by $10 \%$, and ranges about these mean values were reduced by $20 \%$; (2) mean values of $s_{2}-s_{4}$ were increased by $10 \%$, and ranges about these mean values were reduced by $20 \%$; (3) mean values of $s_{2}-s_{4}$ were increased by $20 \%$, and ranges about these values were reduced by $20 \%$; (4) same changes in mean and reduction in ranges as (1), but with a negative correlation $(r=-0.75)$ between egg and brood survival $\left(s_{1}\right.$ and $\left.s_{1}\right)$; and (5) same changes in mean and reduction in range as (2), but with a positive correlation $(r=0.75)$ among $s_{2}, s_{3}$, and $s_{4}$. These adjustments resulted in the vital rates for survival shown in Table A4, which may be compared to those in Table A3.

In addition to these five simulations, we conducted a baseline simulation of no improvements, using our demographic estimates. All of these simulations used a uniform distribution from which to randomly draw vital rates within their estimated ranges. These simulations were conducted as part of Application 2, as described in the Methods.

TABLE A4. Vital rates for survival for the first through fifth simulation for the Greater Prairie Chicken. Table entries are means (ranges).

\begin{tabular}{lccc}
\hline \hline $\begin{array}{l}\text { Vital } \\
\text { rate }\end{array}$ & Simulations 1 and 4 & Simulations 2 and 5 & Simulation 3 \\
\hline$s_{1_{a}}$ & $0.550(0.310-0.790)$ & $\ldots$ & $\ldots$ \\
$s_{1_{b}}$ & $0.385(0.225-0.545)$ & $\ldots$ & $\ldots$ \\
$s_{2}$ & $\ldots$ & $0.490(0.438-0.542)$ & $0.534(0.482-0.586)$ \\
$s_{3}$ & $\ldots$ & $0.561(0.513-0.609)$ & $0.612(0.564-0.660)$ \\
$s_{4}$ & $\ldots$ & $0.312(0.223-0.402)$ & $0.341(0.251-0.430)$ \\
\hline
\end{tabular}

